\title{
Urachal Cyst in a Bitch
}

\section{Fernanda Campos Hertel, Aline Silvestrini da Silva, Gabriela Castro Lopes Evangelista, Amanda Pereira dos Anjos, Andreia Correia Araújo, Evandro Silva Favarato, Tatiana Schmitz Duarte \& Emily Correna Carlo Reis}

\begin{abstract}
Background: The urachus is a tubular structure continuous with the urinary bladder and the allantois in foetal mammals. It serves as a communication between these two structures. At birth, it loses its function and undergoes atrophy by fibrous proliferation within the lumen. When this atrophy process fails at some point, congenital anomalies of the urachus occur. These anomalies are rare in animals, and to our knowledge, the urachal cyst has not yet been described in dogs. The present work is unique in that it reports a case of this congenital defect in a dog. Our aim is to increase awareness and to discuss the clinical presentation, the imaging techniques used, and the final diagnosis of this anomaly.

Case: A 3-year-old bitch Pit Bull was presented for veterinary assistance to investigate recurrent pseudopregnancy and an irregular estrous cycle. The animal presented in good bodily condition, and the white blood count and clinical biochemistry were normal. At ultrasound, 2 tubular structures, filled by an echogenic fluid mimicking uterine topography, were found extending through the umbilical and hypogastric regions. No alterations in structure, echogenicity or echotexture of the other organs were observed, including the ovaries and uterus. On exploratory laparotomy, a cystic structure was found, with 2 segments: the larger one was on the left side, attached to the apex of the bladder by its caudal portion; the other was on the right side, attached to the spleen by its cranial portion and to the apex of the bladder by its caudal portion in connection with the left segment. The ovaries, uterus and uterine horns showed no macroscopic alterations. The structure was removed, and after analysis (macroscopic morphology, wall histopathology and biochemistry of the contained fluid), it was determined to be a urachal cyst. The animal recovered uneventfully.

Discussion: Ultrasound is an important tool for the evaluation of reproductive cycle physiology and its pathologies. At first, the owner's complaint justified a fairly straightforward evaluation and clinical management of pseudopregnancy in a very healthy animal based on the history and clinical and complementary exams. However, ultrasound examination revealed two tubular structures filled with echogenic fluid in the lower abdomen. This made the case more complex, as these were interpreted as uterine horns by three different veterinarians, leading to the possibility of pyometra. The management of this condition must be cautious, since the animal's condition could rapidly deteriorate, necessitating an exploratory laparotomy. Based on the clinical presentation, macroscopic anatomy, fluid biochemistry and histological evaluations, the diagnosis of urachal cyst was proposed. Congenital anomalies occur when the urachus fails to obliterate. Four types can be found: patent urachus, urachal sinus, urachal diverticulum and urachal cyst. The urachal cyst occurs when the urachus encompasses a cyst-like structure that is closed to the umbilicus and the bladder lumen. The urachus becomes a cystic structure since its epithelium is still intact, active and accumulating fluid, like the one analysed in this report. Most urachal cysts in humans remain asymptomatic, but when infected, they can cause focal or diffuse peritonitis and an acute abdomen. Although rare, urachal cysts can develop malignant transformation. The combination of nonspecific symptoms and the infrequent occurrence of this anomaly make its diagnosis a difficult challenge. It is commonly an incidental finding like the case reported here. Therefore, diagnostic imaging techniques, especially ultrasound, are essential for the diagnosis. To our knowledge, this is the first case report of a urachal cyst in a dog. This rare anomaly of the urachus was an incidental finding in the investigation of the reproductive problems of a female dog.
\end{abstract}

Keywords: congenital anomalies, urachal anomalies, urology, ultrasound. 


\section{INTRODUCTION}

The urachus is a tubular structure that is continuous with the urinary bladder and the allantois in foetal mammals. It forms a communication between these two structures $[8,25]$. At birth, it undergoes atrophy by fibrous proliferation within the lumen, and it becomes the medial ligament of the bladder. It loses its foetal function, and the urine is then excreted through the urethra $[1,10,25]$. If this atrophy process fails at some point, congenital anomalies of the urachus occur. In humans, four types of congenital anomalies of the urachus are described: patent urachus, umbilical sinus, urachal diverticulum and the urachal cyst [21].

Among the urachal anomalies, the most frequent one varies between species. In a retrospective study of 103 human patients with urachal anomalies, $36.9 \%$ presented with a urachal cyst, $20.4 \%$ with a patent urachus, $12.6 \%$ with a urachal diverticulum and $10.7 \%$ with a urachal sinus. The remaining $19.4 \%$ were described as having a "nonspecific atretic urachal remnant" in which the entire tract remained as a cord [12]. The veterinary literature cites all four anomalies in animals, but individual case reports were found for urachal diverticulum (the most common type) in dogs $[5,8,16]$ and cattle [10], and patent urachus was reported in cats [1,7] and cattle [9].

The present work describes the occurrence of a urachal cyst in a dog. Our aim is to increase awareness and to discuss the clinical presentation, the imaging techniques used, and the final diagnosis of this anomaly.

\section{CASE}

A bitch Pit Bull, 3-year-old, was presented presented by another service for veterinary assistance at the Veterinary Teaching Hospital of the Federal University of Viçosa. It was investigated the reproductive system due to pseudopregnancy and an irregular estrous cycle, since the owner showed interest in reproduction. Three episodes of pseudopregnancy with 8-month intervals were reported, and flower essences were used as treatment during these episodes.

At clinical examination, vulvar swelling was observed, with an overall good bodily condition. Complementary exams included the erythrogram, white blood count (WBC) and clinical biochemistry including urea, creatinine, ALT, ALP, AST, total protein, albumin, albumin/globulin ratio and glucose. All values were within the normal ranges, except for a slight increase in the ALT (332 IU/L). An abdominal ultrasound was scheduled to evaluate the reproductive system, mainly the ovaries, four days after first attendance.

On ultrasound, 2 tubular structures extending through the umbilical and hypogastric regions were observed. They had the appearance of uterine horns, both with a very thin wall of approximately $0.09 \mathrm{~cm}$, filled by an echogenic fluid (Figure 1A). The structure on the left side presented a diameter of $4.76 \mathrm{~cm}$, and the one on the right side, $2.56 \mathrm{~cm}$. Their extent could not be precisely determined, indicating that the left one extended more cranially than the right one. No alterations in the structure, echogenicity or echotexture of the other organs were observed, including the ovaries (Figure 1C \& D). The uterine body was normal (Figure 1B). A vaginal swab was taken for cytology and analysis of the estrous cycle, distinguishing diestrus from proestrus. The erythrogram, WBC and clinical biochemistry were done, with all values within the normal ranges for dogs. With such a clinical presentation, cystic endometrial hyperplasia, hydrometra and mucometra were considered as possible diagnoses. An exploratory laparotomy was indicated, with a possible ovariohysterectomy; however, due to the animal's good health condition, observation and a return for reevaluation in 3 days was initially recommended, with attention to any behavioural changes.

After 3 days, another ultrasound and complementary exams were performed. The tubular structures were observed with the same characteristics as previously, but with greater diameter $(9.57 \mathrm{~cm}$ on the left and $2.79 \mathrm{~cm}$ on the right). At this point, we decided in favour of an exploratory laparotomy, done on the same day. During the procedure, a cystic structure was found, with 2 segments: the largest one was on the left side, attached to the apex of the bladder by its caudal portion (Figure 2A); the other one was on the right side, attached to the spleen by its cranial portion and to the apex of the bladder by its caudal portion in connection with the left segment (Figure 2B). The spleen was larger than expected, and at the spot where the referred structure was attached, an area similar to a scar with fibrous tissue was observed. The ovaries, uterus and uterine horns were examined, and no macroscopic abnormalities were observed. All spots where the structures attached were evaluated and sutured so that the structure could be removed. It presented a thin wall 
with numerous prominent vessels (Figure 3), and it was filled by a turbid fluid with white lumps. This fluid was analysed for urea, creatinine and protein content, fluid biochemistry (physical, chemical and sediment cytology), cytology after centrifuge and fungal and bacterial cultures and tests of antimicrobial susceptibility. The fluid had a sui generis odor with a $\mathrm{pH}$ of 7.0 and a density of 1.010. No blood, ketone bodies, urobilinogen, bile pigments, bile salts or glucose were present, only proteins $(0.6 \mathrm{~g} / \mathrm{dL})$, urea $(64 \mathrm{mg} / \mathrm{dL})$ and creatinine $(0.6 \mathrm{mg} / \mathrm{dL})$. No cell debris or epithelial cells were observed, and no fungi or bacteria developed in culture. Fragments of the structure wall were collected for histopathology, which showed an epithelium of columnar morphology attached to a loose connective tissue with numerous blood vessels.

Recovery was uneventful, and sutures were removed 10 days after surgery. After 25 days, the owner returned to the Hospital Service with the animal presenting abdominal and inguinal mammary glands producing a milk-like fluid with no signs of infection. The erythrogram and WBC showed no alterations. The prolactin inhibitor metergoline was prescribed $(0.12 \mathrm{mg} / \mathrm{kg}$ twice a day for 5 days $)$, and the animal recovered with no further complications.

\section{DISCUSSION}

As reported by numerous authors and seen in everyday veterinary clinical practice, ultrasound is an important tool for the evaluation of the reproductive cycle physiology and its pathologies $[4,15]$. At first, the owners' complaint warranted a fairly straightforward evaluation and clinical management of pseudopregnancy with a very healthy animal based on the history and clinical and complementary exams. However, the ultrasound examination revealed two tubular structures filled with echogenic fluid in the lower abdomen, making the case more complex, as these were inter-

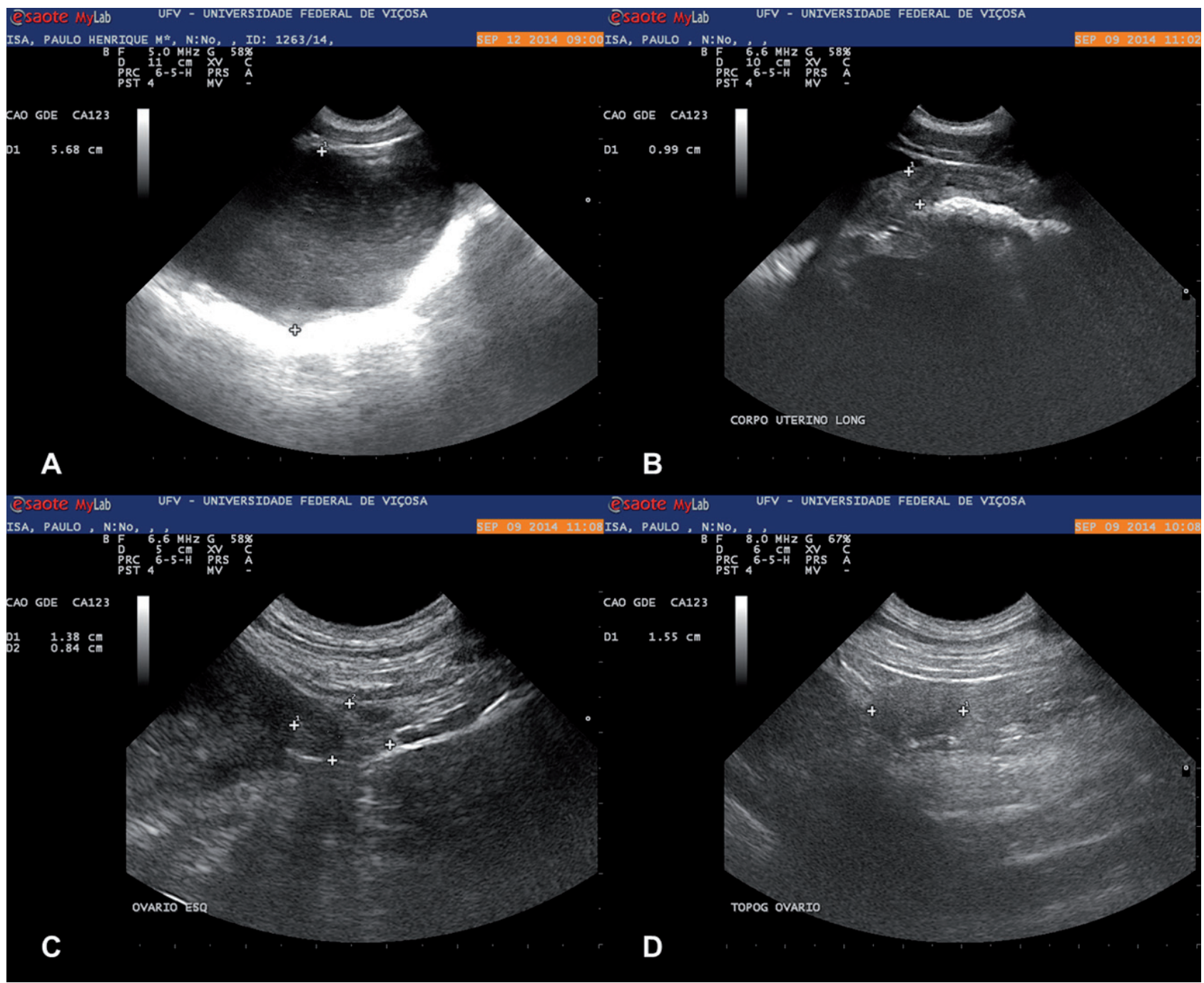

Figure 1. Ultrasound images. A- Transverse sectional image of the tubular structure, showing its diameter (calipers) and the echogenic liquid content. B- Uterus body in a longitudinal section (between calipers). C- \& D- Left and right ovaries (between calipers), respectively. 


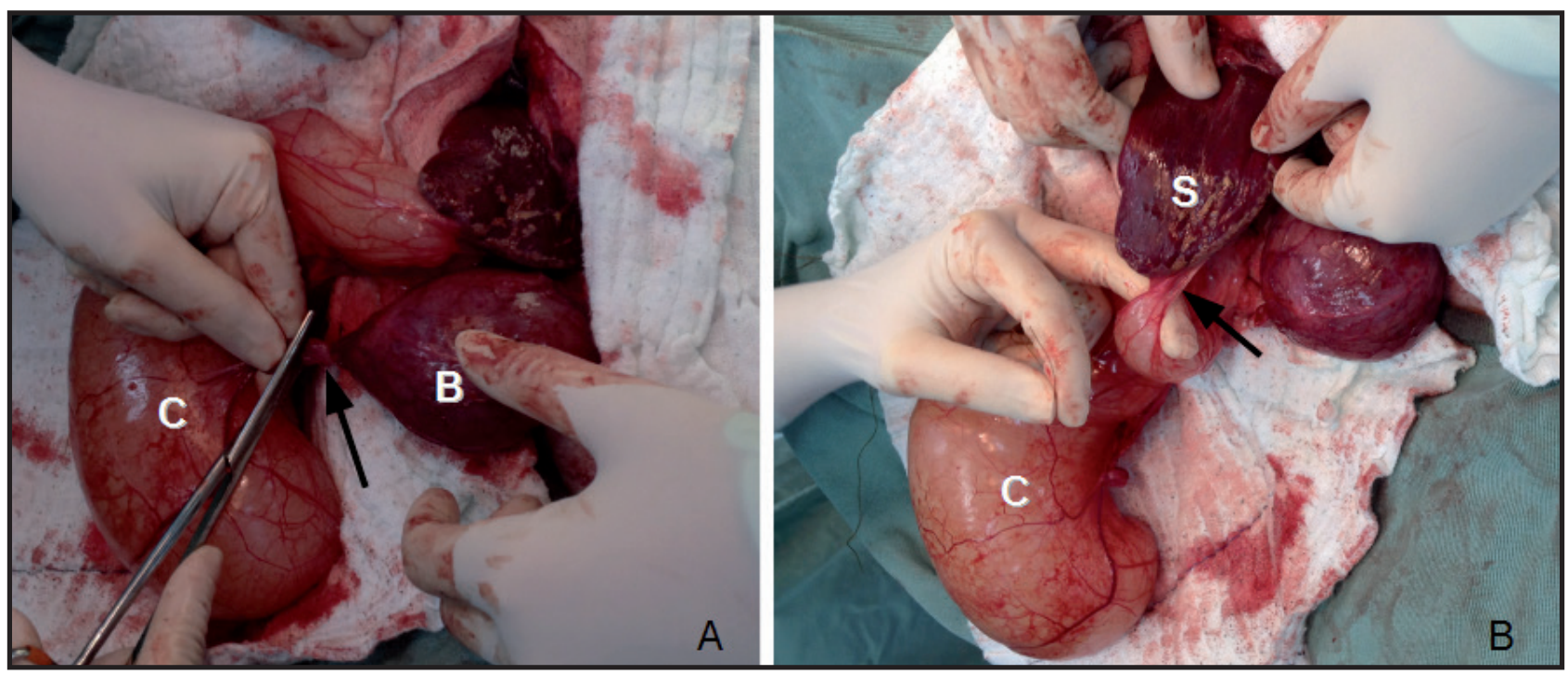

Figure 2. Images during exploratory laparotomy. A- Urachal cyst (C) attached (arrow) to the bladder apex (B). B -Urachal cyst attached to the spleen (S).

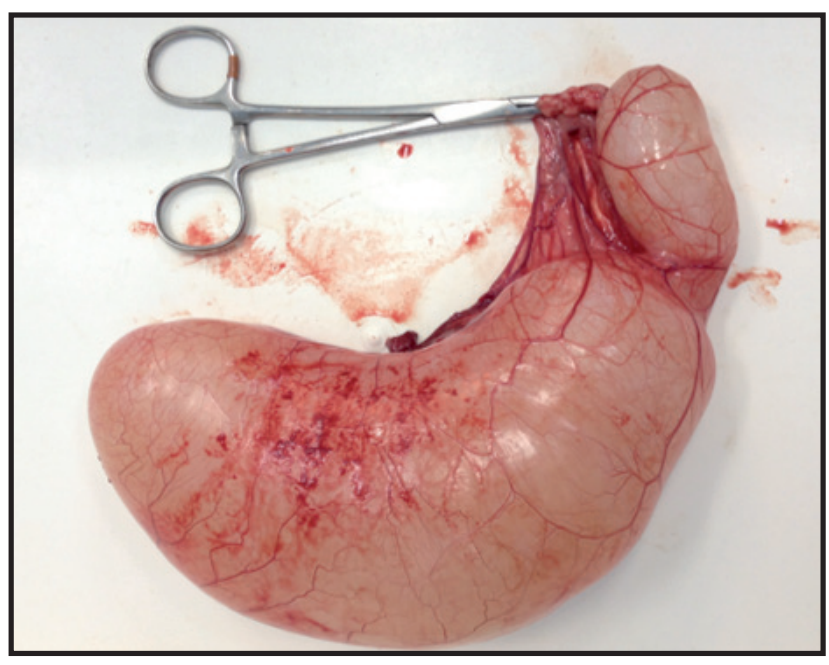

Figure 3. Urachal cyst after surgical removal. Note the tubular morphology and numerous small vessels on its walls.

preted as uterine horns by three different veterinarians. Ultrasound is an important aid for the evaluation of the reproductive system.

Pyometra is the most common condition, characteristically found as an enlarged uterus with tubular horns filled with anechoic or echogenic fluid. Mucometra, haematometra and hydrometra should be suspected when a combination of these ultrasound characteristics is found but no clinical signs consistent with pyometra are identified [15], just as it occurred during the evaluation of the reported case. The management of these conditions must be cautious; with the early detection of a pyometra, before the typical clinical changes occur, or upon infection in one of the other three pathologies, the animal's condition can rapidly deteriorate [4] and necessitate an exploratory laparotomy. Moreover, one could consider a diagnosis of cystic endometrial hyperplasia, but this pathology is characterized by thickened endometrium with cystic structures [20], much different from the thin wall of $0.09 \mathrm{~cm}$ found in this case at ultrasound.

According to the clinical presentation, macroscopic anatomy, fluid biochemistry and histological evaluations, the diagnosis of urachal cyst was made. Urachal congenital anomalies occur when the urachus fails to obliterate. Four types can be found: patent urachus, urachal sinus, urachal diverticulum and urachal cyst [21]. Patent urachus is caused by the total failure of the urachus to obliterate in its entire extension, leaving a communication between the bladder lumen and the exterior of the body. An urachal sinus occurs when the urachus still opens at the umbilicus, but no communication with the bladder still exists [25]. A urachal diverticulum is characterized as a remnant of the urachus forming a bump that opens into the cranial portion of the urinary bladder where urine frequently accumulates, but no communication with the umbilicus exists. The last type is the urachal cyst, in which the urachus encompasses a cyst-like structure, closed to the umbilicus and the bladder lumen, within its length [21]. The urachus becomes a cystic structure similar to the one analysed in this report, because its epithelium is still intact and active, accumulating fluid [10].

Based on the histological characteristics of the structure, another pathology included in the differential diagnosis was hydrosalpinx. However, all the intra-abdominal reproductive organs, namely the uterus, uterine horns and ovaries, were evaluated during the surgery and were considered normal. Additionally, the 
macroscopic anatomy of the structure attached to the bladder dome corroborated the diagnosis of urachal cyst, together with all the other described characteristics. There is still uncertainty regarding the portion of the cyst attached to the spleen. We hypothesise that, due to trauma or another reason, the cyst may have attached to a lesion on the spleen, indicated by the scar observed. Also, there is the possibility of cyst rupture with adhesion to other structures, as previously reported [22,23].

Urachal congenital anomalies are rare in both humans and animals. No individuals reports of a urachal cyst in dogs were found $[5,16]$, but other urachal congenital anomalies in animals have been found, such as patent urachus in cats [1,7], horses [18] and cattle [9] and urachal diverticulum in dogs [8] and bulls [10]. As for humans, the urachal cyst is the most reported urachal anomaly [3,21,25], but it is still a rare condition [3] with possible complications such as infection $[3,11]$.

Most of these urachal cysts in humans develop in the lower third of the urachus. Without infection, they usually remain asymptomatic [6], as in the canine case reported here. When infected, they can cause abdominal pain, fever and leucocytosis. Rupture can occur, leading to focal or diffuse peritonitis and an acute abdomen $[3,6]$. Although rare, malignant trans- formation may occur $[2,14,17]$. Other urachal anomalies are diagnosed in early childhood, needing surgery when bladder infections are recurrent [19].

The combination of nonspecific symptoms and the infrequent occurrence of this anomaly makes its diagnosis a difficult challenge. It is commonly an incidental finding as in the case reported here. The absence of communication with the umbilicus or the bladder lumen makes it difficult to consider urachal anomalies in the differential diagnosis, as no abnormalities on the evaluations of the urinary system or the umbilicus are found. Therefore, diagnostic imaging techniques are essential for the diagnosis, especially ultrasound, computed tomography and magnetic resonance [6]. Ultrasound is the ideal modality for the diagnosis of a urachal cyst in humans [13,21], and Yoo et al. [24] were successful in diagnosing it by ultrasound in $78 \%$ of the cases.

Urachal anomalies are known to be rare in many species. To our knowledge, this is the first case report of a urachal cyst in a dog. It was an incidental finding during an evaluation of the reproductive problems of a female dog expected to breed.

Declaration of interest. The authors declare no conflicts of interest.

\section{REFERENCES}

1 Aleixo G.A.S., Souza M., Mendes Z.F., Baraúna Jr. D., Leite J.E.B., Tenório A.P.M. \& Coelho M.C.O.C. 2007. Persistência do úraco em gato: relato de caso. Arquivo Brasileiro de Medicina Veterinária e Zootecnia. 59(4): 943-947.

2 Brennan K., Johnson P., Curtis H. \& Arnason T. 2019. Urachal mucinous cystic tumor of low malignant potential with concurrent sigmoid colon adenocarcinoma. Case Reports in Gastrointestinal Medicine. 2019: 1434838. https:// doi.org/10.1155/2019/1434838

3 Danial A.K., Tarabishi A.S., Aldakhil A., Alzahran A., Najjar O. \& Ayoub K. 2019. Acute abdomen due to an infected urachal cyst in a 5-year-old female: case report. Journal of Surgical Case Reports. 5: DOI:10.1093/jscr/rjz156.

4 Fayrer-Hosken R.A., Mahaffey M., Miller-Lebl D. \& Caudle A.B. 1991. Early diagnosis of canine pyometra using ultrassonography. Veterinary Radiology. 32: 287-289.

5 Groesslinger K., Tham T., Egerbacher M. \& Lorinson D. 2005. Prevalence and radiologic and histologic appearance of vesicourachal diverticula in dogs without clinical signs of urinary tract disease. Journal of the American Veterinary Medical Association. 226(3): 383-386.

6 Ilica A.T., Mentes O., Gur S., Kocaoglu M., Bilici A. \& Coban H. 2007. Abscess formation as a complication of a ruptured urachal cyst. Emergency Radiology. 13(6): 333-335.

7 Laverty P.H. \& Salisbury S.K. 2002. Surgical management of true patent urachus in a cat. Journal of Small Animal Practice. 43(5): 227-229.

8 Lojszczyk-Szczepaniak A., Smiech A. \& Wojnowski T.A. 2015. Congenital urachal diverticulumin dogs: a case report. Medycyna Weterynaryjna. 66(6): 421-424.

9 Macedo J.T.S.A., Lucena R.B., Giaretta P.R., Kommers G.D., Fighera R.A., Irigoyen L.F. \& Barros C.S.L. 2011. Defeitos congênitos em bovinos da Região Central do Rio Grande do Sul. Pesquisa Veterinária Brasileira. 31(4): 297-306. 
10 Marques L.C., Marques J.A., Marques I.C.S. \& Teixeira M.C.A. 2010. Dilatação cística do úraco e uroperitônio em touros: relato de cinco casos. Arquivo Brasileiro de Medicina Veterinária e Zootecnia. 62(6): 1320-1324.

11 Mrad Daly K., Ben Rhouma S., Zaghbib S., Oueslati A., Gharbi M., Nouira Y. 2019. Infected urachal cyst in an adult: A case report. Urology Case Reports. 26: 100976.

12 Naiditch J.A., Radhakrishbnan J. \& Chin A.C. 2013. Current diagnosis and management or urachal remnants. Journal of Pediatric Surgery. 48(10): 2148-2152.

13 Osbek S.S., Pourbagher M.A. \& Pourbaher A. 1991. Urachal remnants in asymptomatic children: gray-scale and color doppler sonographic findings. Journal of Clinical Ultrasound. 4: 218-222.

14 Prakash M.R., Vijayalaxmi S.V., Maitreyee R.\& Ranjit K.P. 2014. Complex mucinous cystadenoma of undetermined malignant potential of the urachus: a rare case with review of the literature. Malays Journal of Pathology. 36(2): 145-148.

15 Pretzer S.D. 2008. Clinical presentation of canine pyometra and mucometra: A review. Theriogenology. 70(3): 359-363.

16 Remedios A.M., Meddleton D.M., Myers S.L., Outerbridge C.A. \& Arnold P.M. 1994. Diverticula of the urinary bladder in a juvenile dog. The Canadian Veterinary Journal. 35(10): 648-650.

17 Rhudd A., Moghul M., Nair G. \& McDonald J. 2018. Malignant transformation of a urachal cyst-a case report and literature review. Journal of Surgical Case Reports. 2018(3): rjy056.

18 Rocha T.G., Teixeira L.G., Seppa G.S., França T.N. \& Brito M.F. 2007. Fístulas e outras anomalias congênitas dos tratos digestivo e urinário em um potro. Ciência Rural.37(5): 1488-1491.

19 Sato H., Futura S., Tsuji S., Kawase H. \& Kitagawa H. 2015. The current strategy for urachal remnants. Pediatric Surgery International. 31(6): 581-587.

20 Schlafer D.H. \& Giffor A.T. 2008. Cystic endometrial hyperplasia, peseudo-placentational endometrial hyperplasia, and other cystic conditions of the canine and feline uterus. Theriogenology. 70(3): 349-358.

21 Tazi F., Ahsaini M., Khalouk A., Mellas S., Stuurman-Wieringa R.E., Elfassi M.J. \& Farih M.H. 2012. Abscess of urachal remnants presenting with acute abdomen: a case series. Journal of Medical Case Reports. 6: 226-232.

22 Vieira Jr.E.S., Abreu R.A.A. \& Speranzini M.B. 2007. Cisto de úraco em adultos simulando abdômen agudo. Revista do Colégio Brasileiro de Cirurgia. 34(1): 67-68.

23 Wan Y.L., Lee T.Y., Tsai C.C., Chen S.M. \& Chou F.F. 1991. The role of sonography in the diagnosis and management of urachal abscesses. Journal of Clinical Ultrasound. 19(4): 203-208.

24 Yoo K.H., Lee S.J. \& Chang S.G. 2006. Treatment of infected urachal cysts. Yonsei Medical Journal. 47(3): 423-427.

25 Yu J.S., Kim K.W., Lee H.J., Lee Y.J., Yoon C.S. \& Kim M.J. 2001. Urachal remnant diseases: spectrum of CT and US findings. RadioGraphics. 21(2): 451-461. 